\title{
Estudio clínico prospectivo del lente multifocal restor (LMR) implantado de forma unilateral en pacientes sometidos a cirugía de catarata
}

Torres-Bernal LF*, Valdepeña-López Velarde VD**, Rosas-Cabral A*,

Terrones-Saldivar MC ${ }^{* *}$, Schanzlin $D^{* * *}$, Chayet $A S^{* * * *}$

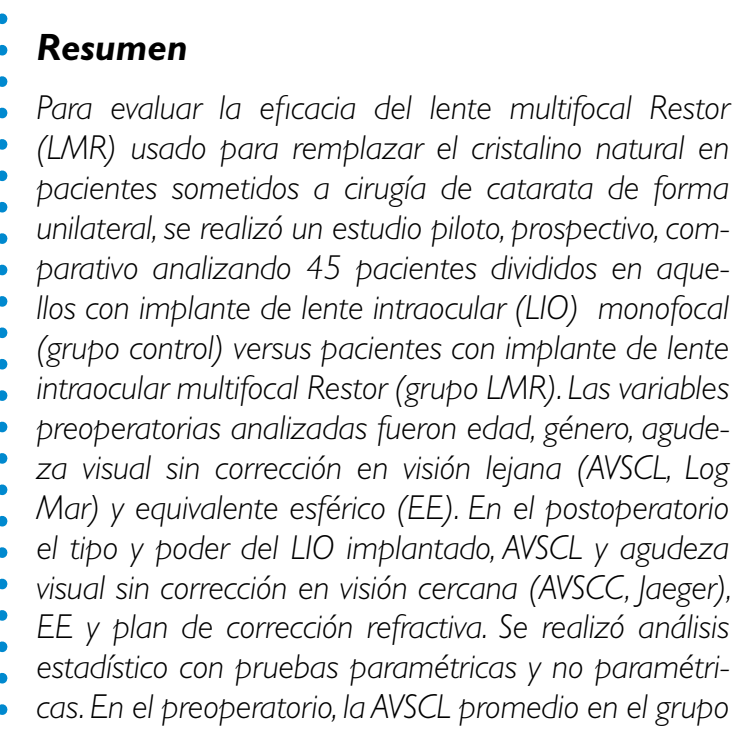

monofocal fue de 1.1 I vs 1.33 (Log Mar) para el grupo multifocal. El EE preoperatorio cambió de -0.37D $a+0.48 D$ en el grupo monofocal, en tanto que en el grupo multifocal cambio de - I. I 8D a 0.14D. La AVSCC postoperatoria fue de Jaeger 10 en el grupo monofocal en tanto que en el grupo multifocal fue de Jaeger I ( $p=$ .0005). El 87.5\% de los pacientes del grupo monofocal uso gafas bifocales en el postoperatorio mientras que el $81 \%$ del grupo multifocal no requirió corrección en visión cercana $(p=0.0000$ I). El LMR implantado de forma unilateral restaura la agudeza visual lejana de forma similar al lente monofocal, con la ventaja de proporcionar una adecuada visión cercana de forma simultanea. El $80 \%$ de los pacientes con LIO multifocal unilateral logran la independencia del uso de anteojos a diferencia de lo pacientes con el LIO monofocal. LUXMÉDICA, 2011;6(17):

Palabras clave: lente multifocal, catarata, Restor

* Profesores investigadores del Centro de Ciencias de la Salud.UAA

** Pasante de Servicio Social, CCS, UAA

*** Investigador del Shiby Eye Center UCSD,Sn Diego, CA, EUA

**** Investigador del CODET Vision Institute, Tijuana, Baja California

Fecha de recibido 1 noviembre 2010

Fecha de aceptación 2 diciembre 2010

Correspondiencia Dr en C Luis Fernando Torres Bernal, Centro de Ciencias de la Salud, UAA. Av Universidad \#940 Ciudad Universitaria CP 20100 Ags, tel 9108440 Correo electrónico ftorresb@yahoo.com. 


\section{Introducción}

La meta del cirujano contemporáneo de catarata es la búsqueda de la perfección visual. El paciente operado de catarata debe obtener un resultado visual óptimo y rápido, con una adecuada visión lejana, una aceptable visión cercana y un resultado refractivo cercano a la emetropía, logrando la independencia del uso de anteojos ${ }^{1}$. Para obtener este resultado se requiere de un cálculo exacto del poder de la lente intraocular (LIO) en el preoperatorio junto con un procedimiento de extracción de catarata sin complicaciones donde además el astigmatismo preexistente sea corregido².

En la actualidad la extracción de catarata se realiza mediante facoemulsificación, técnica que consiste en la fragmentación del cristalino utilizando energía ultrasónica, a través de una incisión que suele medir aproximadamente $2.8 \mathrm{~mm}^{3}$. Posteriormente se introduce un lente intraocular el cual sustituye el poder refractivo del cristalino. El primer LIO implantado en el ojo humano fue diseñado por Sir Harold Ridley, con el objetivo de corregir la afaquia unilateral posterior a la extracción de la catarata en un intento por restaurar la visión binocular ${ }^{4}$. Sin embargo, Ridley comprendió que un ojo pseudofáquico era incapaz de acomodar. Desde entonces se reconoce que los LIO's monofocales son excelentes para la restauración de la visión de lejos, con el inconveniente de que el paciente debe de usar anteojos para restaurar su visión cercana o de lectura ${ }^{5}$.

En la última década el avance de la tecnología ha dado origen a diversas modificaciones en el diseño de lentes intraoculares ${ }^{6}$. Así pues, han surgido nuevos lentes que ofrecen mejorar la calidad de la visión en pacientes pseudofacos ${ }^{7}$. Los lentes multifocales intentan conseguir el máximo acercamiento a la visión fisiológica del paciente ${ }^{7}$, surgiendo como alternativa a otros procedimientos como la monovisión, sin embargo se ha pregonado el uso bilateral necesario para que el lente cumpla su función. Sin embargo algunos autores han implantado los lentes mutifocales de forma unilateral ${ }^{8}$.

El lente intraocular multifocal AcrySof Restor (LMR, Alcon, Texas, EUA), es un lente diseñado para alcanzar una agudeza visual satisfactoria lejana, cercana y disminuir la dependencia al uso de lentes ${ }^{9}$. Este lente de acrílico, hidrofóbico, que presenta un diseño especial, con una óptica biconvexa en donde la superficie anterior es apodizado y difractiva, con anillos concéntricos en los 3,6 mm centrales, con pasos de altura que van disminuyendo de 1,3 $\mu$ en la zona central a $0.2 \mu$ en la periferia, lo que ayuda a distribuir la luz en una gama completa para la visión ${ }^{5},{ }^{10-13}$. Diversos estudios han demostrado que este lente multifocal implantado bilateralmente logra una mejor agudeza visual cercana que los LIO's monofocales, con una visión de lejos similar a la obtenida con los LIO's monofocales pero mejorando la visión cercana sin ningún tipo de efecto adverso importante en visión lejana ${ }^{6}$. A pesar de las ventajas potenciales 
de las lentes multifocales, las lentes monofocales siguen siendo el tipo de lentes más empleado en la cirugía de catarata, probablemente por el costo de los primeros y además por las exigencias que implica para el cirujano, implantar estos modelos ${ }^{14-15}$. Actualmente existen escasos reportes sobre el implante unilateral con este tipo de lentes. Estos se preguntan si la compensación propia de un LIO multifocal da lugar a una mejor, igual o menor función visual en comparación con el LIO monofocal. La pregunta clave que todos buscan contestar es si ópticamente, existe una mejoría visual objetiva y subjetiva después de la cirugía de catarata, y que los resultados sean más elevados que los ya conocidos o esperados por un LIO monofocal 6,15,20-21. El objetivo del presente estudio es el de evaluar la efectividad del LMR usado para remplazar el cristalino natural en pacientes sometidos a cirugía de catarata de forma unilateral.

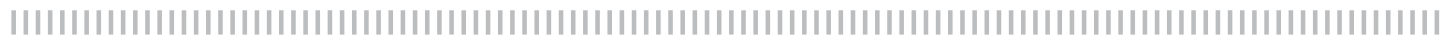

\section{Material y métodos}

Se realizó un estudio piloto, prospectivo y comparativo con la finalidad de establecer un concepto inicial del LMR en su uso unilateral en la clínica. Dado que se trata de un estudio piloto, no se requirió de un estimado del tamaño de muestra formal, dándose ésta por conveniencia. Se incluyeron 24 pacientes que recibieron LIO monofocal (Acrysoft, Alcon) de forma unilateral y 21 pacientes a los que se les implantó de forma unilateral un lente intraocular apodizado multifocal (LMR, Restor, Alcon). Se incluyeron pacientes que reunieron los criterios de inclusión siendo mayores de edad, de cualquier género, sometidos a facoemulsificación no complicada, con implante de lente intraocular de una pieza, plegable, de acrílico hidrofóbico en bolsa capsular monofocal o multifocal, sin patología ocular asociada. Se excluyeron todos aquellos pacientes que no otorgaron su consentimiento informado, que presentaron laxitud zonular, pseudoexfoliación, patología de segmento anterior asociada, enfermedades retinianas degenerativas, que requirieron tratamiento retiniano con láser, que presentaban glaucoma o hipertensión ocular y aquellos pacientes con complicaciones durante la cirugía de catarata como rexis incompletas, pérdida de vítreo, trauma iridiado, complicaciones corneales o ruptura zonular o capsular. Se eliminaron a aquellos pacientes que no cumplieron con la evaluación postoperatoria. Para todos los casos se realizó cálculo de LIO usando un ecógrafo modo A de inmersión Alcon, Ocuscan usando un método de inmersión realizado por el mismo operador en todos los casos. Las queratometrías usadas para el mismo fueron tomadas usando el topógrafo corneal de la compañía Oculus, Inc. Todos los pacientes con astigmatismos mayores a 1.0 D en el grupo de multifocal fueron sometidos a incisiones relajantes corneales con el fin de mejorar su refracción postoperatoria. Las variables preoperatorias analizadas fueron edad, género, agudeza visual sin corrección de lejos (AVSCL) y equivalente esférico (EE). En el período postquirúrgico se analizó el tipo y poder del LIO implantado, AVSCL, agudeza visual sin corrección de cerca (AVSCC), EE y plan de manejo refractivo postoperatorio. Se realizó un análisis estadístico con pruebas paramétricas y no paramétricas. 


\section{Resultados}

La edad promedio de los pacientes del grupo de LIO monofocal fue de 71.29 años, mientras que la de los pacientes del grupo de lente multifocal fue de 58.9 años. Con respecto al género el $75 \%$ de los pacientes del grupo monofocal fueron hombres mientras que la mayoría de los pacientes del grupo multifocal fueron mujeres (52.40\%) (Tabla 1).

\section{Tabla I}

\section{Variables demográficas de los grupos de estudio.}

\begin{tabular}{|lcc|}
\hline Variables demográficas & \multicolumn{2}{c|}{ Grupo de estudio } \\
\hline & MONOFOCAL & MULTIFOCAL \\
\hline PROMEDIO DE EDAD (años) & 71.29 & 58.9 \\
\hline RANGO (años) & $(44-88)$ & $(26-76)$ \\
\hline MASCULINO (\%) & 75 & 47.6 \\
\hline FEMENINO (\%) & 25 & 52.4 \\
\hline
\end{tabular}

La agudeza visual sin corrección promedio del grupo monofocal fue de 1.11 (Log Mar) y su equivalente esférico preoperatorio fue de -0.37D. A todos los pacientes del grupo monofocal se les implantó de forma unilateral un lente Acrysoft tipo SA60AT o SN60AT; el poder promedio del LIO implantado fue de 20.54 dioptrías. En el período postoperatorio, la agudeza vi- sual de lejos promedio fue de 0.20 (Log Mar) y de cerca fue de Jaeger 10. Su capacidad visual de lejos tuvo una media de 0.15 , con equivalente esférico promedio de 0.48. El plan de manejo postoperatorio en el $87.5 \%$ de los pacientes, incluyó el uso de lentes bifocales, mientras el $12.5 \%$ de los pacientes no requirió el uso de ellos (Tabla 2 y figura 1).

\section{Tabla 2}

Variables refractivas de los grupos de estudio.

\begin{tabular}{|c|c|c|c|}
\hline \multirow[t]{2}{*}{ Variables refractivas } & \multicolumn{3}{|c|}{ Grupo de estudio } \\
\hline & MONOFOCAL & MULTIFOCAL & $\mathrm{p}$ \\
\hline PODER LIO (D) & 20.54 & 20.97 & \\
\hline AVSC LEJOS PREOPERATORIA (Log Mar) & 1.11 & 1.33 & 0.364 \\
\hline AVSC LEJOS POSTOPERATORIA (Log Mar) & 0.2 & 0.11 & 0.060 \\
\hline AV CERCA POSTOPERATORIA (Log Mar) & $J=10$ & $J=1$ & 0.000 \\
\hline EE PREOPERATORIO (D) & -0.37 & -1.18 & 0.435 \\
\hline EE POSTOPERATORIO (D) & 0.48 & 0.14 & 0.357 \\
\hline
\end{tabular}

$L I O=$ Lente Intraocular, $D=$ Dioptrías, Log Mar= Logaritmo del ángulo de resolución mínima, AVSC= Agudeza Visual Sin Corrección, EE = Equivalente Esférico 
Tipo de corrección visual postoperatoria

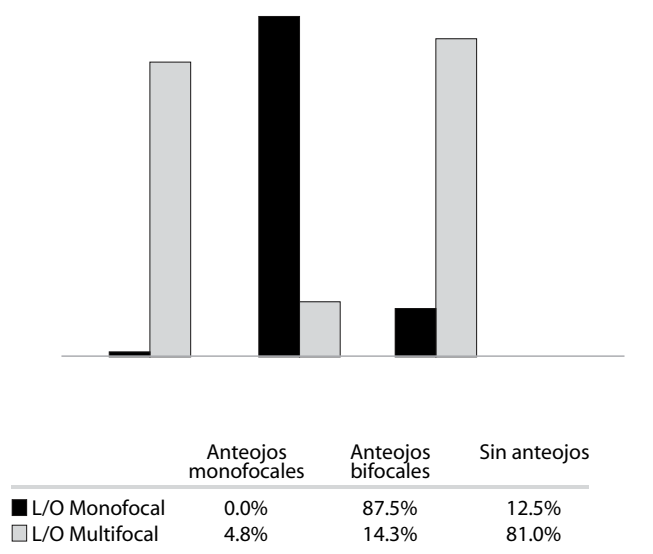

Figura 1. Tipo de corrección visual postoperatorio.

La agudeza visual sin corrección del grupo multifocal fue de 1.33 (Log Mar) y su equivalente esférico preoperatorio fue de -1.18D. A todos los pacientes del grupo multifocal se les implantó de forma unilateral un lente multifocal apodizado (Alcon, Labs, Restor); el poder promedio del lente intraocular implantado fue de 20.97 dioptrías. En el periodo postoperatorio, la agu- deza visual de lejos promedio fue de 0.11 (Log Mar) y de cerca de Jaeger 1. Su capacidad visual de lejos tuvo una media de 0.01 , con equivalente esférico promedio de 0.14 .El plan de manejo postoperatorio fue el $81 \%$ de los pacientes no necesitaron corrección óptica ni para visión de lejos ni de cerca, mientras el $19 \%$ requirió el uso de lentes de armazón. (Tabla 2 y figura 1 )

\section{| | | | | | | | | | | | | | | | | | | | | | | | | | | | | | | | | | | | | | | | | | | | | | | | | | | | | | | | | | | | | | | | | | | | | | | | | | | | | | | | | | | | | | | | | | | | | | | | | | | | | | | | | |}

\section{Discusión}

La cirugía de catarata ha sido revolucionada en los últimos cinco años con el advenimiento de nuevas tecnologías que permiten realizar una cirugía más controlada, con mayor éxito visual y una recuperación más rápida. Lo anterior, junto con la creación de nuevos diseños de los lentes intraoculares permite ofrecer a los pacientes no solo una buena visión lejana sino también una mejor visión cercana ${ }^{3}$.

Nuestro estudio muestra diferencias clínicas esperadas entre los dos grupos, lo que comprueba la eficacia del LMR. En cuanto a los datos demográficos, los pacientes con implante de lente intraocular multifocal (grupo Restor) son una década menor que los pacientes del grupo monofocal, esto se debe probablemente, a que la gente más joven tiene, tanto expectativas como exigencias visuales mayores en el postoperatorio, y por lo tanto buscan atención médica de forma más temprana y solicitan un lente multifocal, a diferencia de una persona de mayor edad, en quienes sus actividades cotidianas han cambiado desenvolviéndose en un ámbito de menor exigencia visual. De la misma forma, y en cuanto al género, nos damos cuenta que el grupo de pacientes con LIO multifocal en su mayoría son mujeres, esto puede deberse a que tienen mayores exigencias cosméticas, no solo toca el aspecto visual sino el de lograr la independencia del uso de anteojos tanto para ver de cerca como de lejos.

En los dos grupos existió una mejoría tanto de la agudeza visual sin corrección como en la agudeza visual mejor corregi- 
da o capacidad visual para ver de lejos. Sin embargo se demostró una diferencia clínica aunque no estadísticamente significativa en cuanto a que el grupo de pacientes con LIO multifocal presentó una línea visual más de mejoría, en comparación con el grupo de LIO monofocales. De la misma manera el equivalente esférico se acercó más a la emetropía en el grupo de LIO's multifocales que en el de monofocales. Esto puede explicarse como consecuencia del uso de técnicas quirúrgicas adyuvantes como la incisiones relajantes limbares o corneales que corrigen el astigmatismo corneal transoperatorio, las cuales se usaron únicamente en el grupo de los lentes multifocales.

Los resultados mostraron la eficacia del LIO multifocal al implantarse de forma unilateral, dado que proporciona una visión óptima de lejos y de cerca. Esta mejoría en cuanto a la agudeza visual de cerca, no fue alcanzada en el grupo de LIO's monofocales, lo que conlleva a que más del $85 \%$ de los pacientes requieran el uso de gafas en el postoperatorio. Por otra parte, mas del $70 \%$ de los pacientes del grupo multifocal unilateral, no necesitó el uso de lentes bifocales. Hay dos tipos básicos de tecnología multifocal existente: difractiva y refractiva. Un estudio teórico mostró que el lente intraocular multifocal difractivo es superior al lente intraocular multifocal refractivo para visión cercana, considerando que para la visión lejana ambas lentes son comparables ${ }^{9}$. Estudios clínicos confirman las ventajas de la lente difractiva en comparación de la refractiva principalmente en visión cercana al implantarse de forma bilateral ${ }^{8,11-15,4-5}$. Se ha demostrado que las lentes intraoculares multifocales refractivas son significativamente más pupilo dependientes ${ }^{5,11-13}$. Un diámetro pupilar $<4.5 \mathrm{~mm}$ no puede proveer una $\mathrm{AV}$ útil a lo cerca ${ }^{19}$. Por lo tanto, el tamaño pupilar en poblaciones con cataratas, es una consideración importante en la selección de candidatos para este lente intraocular multifocal. Por lo tanto, sobresale el hecho de que este nuevo lente multifocal (LMR) tiene la capacidad de enfocarse en varias distancias, lo cual permite restaurar la visión lejana y cercana y evitar el uso de lentes de armazón en el postoperatorio en un gran porcentaje de los casos implantados de forma unilateral. Este hecho contrasta con la literatura mundial en donde se indica que es necesaria el implante bilateral del lente multifocal para que los pacientes logren la independencia del uso de anteojos 8,11-15,4-5. Asimismo abre nuevas expectativas para aquellos pacientes que tienen un mayor demanda visual de lejos pero que asimismo requieren su visión cercana para realizar actividades cotidianas básicas sin usar lentes, como el leer la portada de una revista, o el leer las etiquetas de un producto en un supermercado, lo que se traduce en una mayor comodidad y calidad de vida del paciente.

\section{Conclusiones}

El lente multifocal apodizado (LMR) es eficiente al restaurar la agudeza visual de lejos de forma similar al lente monofocal, con la ventaja de proporcionar una adecuada visión cercana de forma simultánea, aún al implantarse de forma unilateral. Estos resultados permiten la independencia del uso de anteojos en el periodo postoperatorio de hasta el $80 \%$ de los pacientes implantados de forma unilateral con lentes multifocales. 


\section{Bibliografía}

1 Steinert RF. Visual outcomes with multifocal intraocular lenses. Curr Opin Ophthalmol. 2000; 11:1221.

2 H.V. Gimbel, D.R. Sanders and M.G. Raanan, Visual and refractive results of multifocal intraocular lenses, Ophthalmology 98 (1991), pp. 881-887 discussion by JT Holladay, 888.

3 Chayet Arturo MD, Sancho Cristhian MD, Torres Luis MD, PhD. Procedimientos Refractivos antes y durante la cirugía de catarata. Ajuste del resultado al objetivo, Buscando la excelencia en la cirugía de catarata. Edit. Glosa. Primera edición. España, 2006. Pág.: 337-346. ISBN 84-7429-284-0.

4 Ridley $\mathrm{H}$. Intra-ocular acrylic lenses. Trans Ophthalmol Soc UK. 1951; 71:617-621.

5 Werner L, Olson RJ, Mamalis N...New technology IOL optics. Ophthalmol Clin North Am. 2006 Dec; 19(4):469-83. Review.

6 Martin Leyland, Edoardo Zinicola, MD. Multifocal versus monofocal intraocular lenses in cataract surgery: A systematic review. Volume 110, Issue 9, Pages 1789-1798 (September 2003).

7 Pieh S, Marvan P, Lackner B, et al. Quantitative performance of bifocal and multifocal intraocular lenses in a model eye: point spread function in multifocal intraocular lenses. Arch Ophthalmol. 2002; 120:2328.

8 Weghaupt H, Pieh S, Skorpik C. Comparison of pseudoaccommodation and visual quality between a diffractive and refractive multifocal intraocular lens. J Cataract Refract Surg. 1998; 24:663-665.

9 Souza CE, Muccioli C, Soriano ES, Chalita MR, Oliveira F, Freitas LL, Meire LP, Tamaki C, Belfort R Jr.. Visual performance of Acrysoft Restor apodized diffractive IOL: a prospective comparative trial. Am J Ophthalmol. 2006 May; 141(5):827-832. Epub 2006 Mar 20.

10 Steinert RF, Aker BL, Trentacost DJ, et al. A prospective comparative study of the AMO ARRAY zonalprogressive multifocal silicone intraocular lens and a monofocal intraocular lens. Ophthalmology. 1999; 106:1243-1255.

11 J.T. Holladay, H. van Dijk and A. Lang et al., Optical performance of multifocal intraocular lenses, J Cataract Refract Surg 16 (1990), pp. 413-422 erratum, 781.
12 Walkow T, Liekfeld A, Anders N, et al. A prospective evaluation of a diffractive versus a refractive designed multifocal intraocular lens. Ophthalmology. 1997; 104:1380-1386.

13 Nowak MR, Strobel J, Jacobi F. Glare and contrast with diffraction intraocular lenses [in German]. Fortschr Ophthalmol. 1991; 88:125-127.

14 R.J. Duffey, R.W. Zabel and R.L. Lindstrom, Multifocal intraocular lenses, J Cataract Refract Surg 16 (1990), pp. 423-429.

15 37Gimbel HV, Sanders DR, Raanan MG. Visual and refractive results of multifocal intraocular lenses. Ophthalmology. 1991; 98:881-888.

16 Apple DJ, Peng Q, Visessook N, et al. Eradication of posterior capsule opacification (Documentation of a marked decrease in $\mathrm{Nd}$ :YAG laser posterior capsulotomy rates noted in an analysis of 5416 pseudophakic human eyes obtained postmortem). Ophthalmology. 2001; 108:505-518.

17 R.J. Uusitalo, T. Brans, T. Pessi and A. Tarkkanen, Evaluating cataract surgery gains by assessing patients' quality of life using the VF-7, J Cataract Refract Surg 25 (1999), pp. 989-994.

18 E.P. Steinberg, J.M. Tielsch and O.D. Schein et al., The VF-14; an index of functional impairment in patients with cataract, Arch Ophthalmol 112 (1994), pp. 630-638.

19 E.P. Steinberg, J.M. Tielsch and O.D. Schein et al., National study of cataract surgery outcomes; variation in 4-month postoperative outcomes as reflected in multiple outcome measures, Ophthalmology 101 (1994), pp. 1131-1140 discussion by DM O'Day, 1140-1141.

20 R.F. Steinert, C.T. Post Jr. and S.F. Brint et al., A prospective, randomized, double-masked comparison of a zonal-progressive multifocal intraocular lens and a monofocal intraocular lens, Ophthalmology 99 (1992), pp. 853-860 discussion by JT Holladay, 860861.

21 Javitt JC, Steinert RF. Cataract extraction with multifocal intraocular lens implantation: a multinational clinical trial evaluating clinical, functional, and quality-of-life outcomes. Ophthalmology. 2000; 107:2040-2048 